\title{
Promoting New Patterns in Household Energy Consumption with Pervasive Learning Games
}

\author{
Magnus Bang, Anton Gustafsson, and Cecilia Katzeff \\ Interactive Institute, Eskilstuna, Sweden \\ \{magnus.bang, anton.gustafsson, cecilia.katzeff\}@tii.se
}

\begin{abstract}
Engaging computer games can be used to change energy consumption patterns in the home. PowerAgent is a pervasive game for Java-enabled mobile phones that is designed to influence everyday activities and use of electricity in the domestic setting. PowerAgent is connected to the household's automatic electricity meter reading equipment via the cell network, and this setup makes it possible to use actual consumption data in the game. In this paper, we present a two-level model for cognitive and behavior learning, and we discuss the properties of PowerAgent in relation to the underlying situated learning, social learning, and persuasive technology components that we have included in the game.
\end{abstract}

\section{Introduction}

Computer games have been used as educational tools for over two decades. However, the efficacy of this learning and behavior-modifying approach has been criticized on several accounts. For example, researchers are questioning whether students can actually use the knowledge they acquire in simulation games and apply it to real-world tasks (e.g., generalization and transfer of knowledge) [1,2]. Moreover, traditional interactive games seem to result in shallow learning. This problem has to do with difficulties in conveying deeper meaning, as well as the underlying learning models in the expeditious interactive games [3]. Modern theories on learning have also criticized the notion of knowledge transfer. Instead, researchers emphasize the importance of relevant and authentic real-world tasks in learning, and they discuss the social aspects of education, including mediation of skills via peer interactions [4,5]. Among others, Brown and colleagues [4] have stated that learners actively construct knowledge, and that it is built on previous experience of real-world tasks. Studies in the field of persuasive technology have also highlighted the role of social aspects such as peer group pressure [6,7].

Advances in augmented reality and ubiquitous computing show promise, and new computer games in this area have the potential to turn ordinary surroundings into sophisticated learning environments. Pervasive games constitute a relatively new class of computer games that extend the gaming experience to the real world [8]. In these games, the players are framed by real-life surroundings and interact with computerenhanced objects. This approach has attractive properties from the perspective of 
learning and behavioral modification: learners do not have to rely on simulation, and tasks can be trained in the real environment, which might reduce problems with knowledge transfer.

According to Thomas [9], pervasive learning games are important primarily because they entail social processes that connect learners with communities of devices, people, and real learning situations. We are exploring this new game approach to target energy-use behaviors in the home. The hypothesis underlying our work is that persuasive games have the potential to strengthen situated learning and promote behavioral changes by reframing familiar activity and social systems.

Few pervasive games have been developed for the purpose of learning [ibid.]. Furthermore, to our knowledge, no games in this genre have been described that promote behavioral changes aimed at altering energy usage patterns. In this paper, we present PowerAgent, a pervasive learning game for teenagers designed to encourage more efficient energy use in the home. To strengthen the learning and persuasion components, PowerAgent has been constructed from the perspectives of situated learning [5] and social learning theory ${ }^{1}[10,11]$, and it also makes use of persuasive technology methods [6]. We begin by discussing the area of pervasive gaming and summarizing research on pervasive learning games. Thereafter, we present the theoretical foundation on which our game rests. The main part of the text covers the design of the game, or, more correctly, how the micro-social activity system is reshaped by the game. Lastly, we consider aspects of the design of pervasive learning games from the standpoint of behavioral and persuasion technology.

\section{Pervasive Gaming and Learning}

Pervasive games belong to a rather heterogeneous category of such entertainment that includes location-based games, urban mobile games, cross-media or hybrid games, immersive games, urban super-hero games, and alternative reality games [9]. The main feature that distinguishes pervasive games from traditional simulation games is that they extend the gaming experience to the physical world [8]. Consequently, there is no need for developers to simulate a game world such as that in the Sims [12]; instead, the approach involves overlay of game scenarios onto real world objects and environments (cf., augmented reality). Typically, the games utilize technologies like ad hoc computer networks and satellite positioning to link and track devices and users in the physical surroundings. Special interfaces such as displays and cameras are frequently used to superimpose the virtual game world and its tasks onto everyday physical artifacts and spaces. BotFighters [13] was an early location-based game on mobile phones, the mission of which was to find and defeat other players' bots out on the streets. By comparison, the goal of the modern pervasive game called Epidemic Menace [14] is to seek out and destroy viruses that invade different physical locations. To enhance the gaming experience, Epidemic Menace includes a set of special interfaces and physical props, such as mobile phones, mobile-augmented reality systems, physical game boards, and special communication stations.

1 In 2001, Bandura renamed social learning theory (SLT) social cognitive theory to emphasize the cognitive aspects of learning and behavior modification. 
Pervasive games are particularly interesting when considered from the perspective of persuasive technology and learning. Researchers such as Sotamaa [15] have argued that a fundamental trait of pervasive games is that they alter the social landscape. A possible consequence of blurring the distinction between different social landscapes is that the internal social rules of a game can have an influence on previously established activity rules. That is, activities in the game have the potential to be learned and transferred to related off-game activities. Of interest in this discussion are the four tenets of pervasive learning games suggested by Thomas [9]: community, autonomy, locationality, and relationality:

- Community: Pervasive learning is a social process that connects learning to communities of devices, people, and situations that can also include other pervasive learning situations. Learning in such environments are mediated by peers in the game community.

- Autonomy: Gamers direct learning experiences themselves and are in control of their learning processes.

- Locationality: Learning occurs at places and at times that are relevant for the learner.

- Relationality: Learners construct meaningful and relevant learning situations to which they can relate. Learning familiar concepts within their own personal environment allows them to better understand the implications of what they have learned, and this facilitates the construction of meaning.

Before we describe the PowerAgent game, it is appropriate to discuss underlying theories of learning that are related to our design.

Modern theories of learning seem to involve a consensus of major standpoints. First, as pointed out above, the learner is regarded as an active constructor of meaning, which is a view of learning emphasized within constructivist theory [16]. Second, learning is regarded as a social process; just as the physical world is shared by all of us, we also share an understanding of it [17]. Learning is a process that is in progress not only in the individual learners, but also in the culture of the learners [5]. For example, according to ideas of situated learning [4] and distributed cognition [18], knowledge exists not only within individuals, but also in the discourse among those persons, their interconnecting social relations, and the physical artifacts they employ, as well as the theories, models, and methods they use to produce supporting tools.

Social learning theory (SLT) is related to the above-mentioned perspectives, ${ }^{2}$ in particular situated action [10]. SLT is especially attractive for the purpose of persuasive game design, because it can provide principles to guide that process. For instance, SLT includes behavioral reinforcers such as positive feedback, which is very

\footnotetext{
${ }^{2}$ Situated learning, situated action theory, distributed cognition, and social learning theory share several assumptions about how learning is achieved. However, it should also be said that there are significant divergences on some issues, particularly in terms of the feedback components and modeling that are not addressed in the situated theories.
} 
common in interactive games, and such notions are outside the scope of the situated learning theories. The power of SLT lies in that it explains human behavior as an interaction process between cognitive, behavioral, and social/environmental components.

The basic tenet of SLT is the importance of observing and modeling: people learn by observing the behavior of others and by watching the outcome of actions (cf., vicarious learning). The outcome of observations can be in the form of cognitive learning alone, and it can also entail changes in behavior. The learnt is subsequently reinforced by several factors. For example, imitating the model can result in being accepted in the social group from which the model originated. Compliments and praise from people that back such behavior also reinforce the modeled behavior. Vicarious reinforcements, that is, when the model is being reinforced and that is observed by the learner, can increase the response and thus be regarded as yet another form of reinforcement.

According to Bandura, there can be two models, one that is symbolic and one that is live. The latter refers to a person who demonstrates behavior in the real world, and the former can be a person portrayed in a medium such as television and computer programs. It should also be noted that the maximum level of observational learning is achieved when the modeled behavior is symbolically rehearsed and the behaviors are subsequently enacted and rehearsed in the real world. Moreover, symbolic coding by use of words, labels, and images can further strengthen retention of behaviors.

\section{PowerAgent}

PowerAgent is a pervasive game for teenagers designed to have a positive influence on everyday energy consumption behavior in the home. It can be seen as a hybrid pervasive game, because it makes use of several media components - both traditional gaming and pervasive mechanisms - to support both cognitive and behavioral learning. A key design goal was to foster social interactions with peers that are playing the game and also with family members that becomes entangled indirectly in the game due to its pervasive social nature. PowerAgent is shown in Figure 1.

\subsection{Game Scenario}

The person playing PowerAgent has the role of a secret agent, and the mobile phone is the main agent tool. Via the phone, the boss, the mysterious Mr. Q, gives the player special missions to save the planet from the energy crisis. These are divided into training missions and real-world tasks. The training missions are played on the cell phone and these precede the actual real-world missions in the home. This training element is in the form of a traditional platform game characterized by jumping/ climbing to and from suspended platforms (cf., Super Mario Bros.), which is done to catch batteries. Every battery contains a suggestion on how to act in order to be energy efficient in the upcoming real-world task, and it also holds information on how to influence family members. 


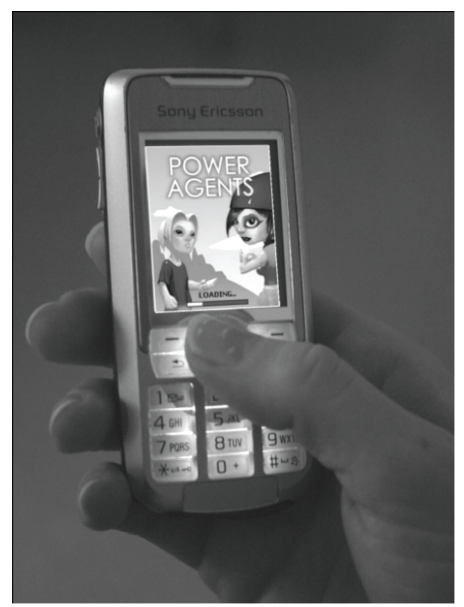

Fig. 1. The PowerAgent game

For example, the tips for the cooking food mission can be the following: "Use the microwave oven instead of the ordinary oven, use the water boiler to heat water instead of putting the kettle on the stove, and tell everyone that you love food cooked in the microwave."

All real-world missions are focused on saving electricity in the home, particularly in conjunction with everyday activities. Typical missions include (1) adjusting the heating in the house, (2) washing clothes, (3) cooking food, (4) switching off all standby appliances, and (5) minimizing the total household energy use for a day. Hence, the goal is to figure out how to reduce energy consumption while performing the mission assigned by Mr. Q. The mobile phone is connected to special equipment in the home that measures the use of electricity and heat water during the missions. In this way, it is possible for us to provide feedback on the success of the missions and reinforce behaviors that are appropriate from the standpoint of energy conservation.

PowerAgent is normally played by two teams with five members each. The realworld tasks are done individually at home, but at the end of each mission the results from all team members are summarized and compared with the results of the competing team (see Fig. 3g). We included the competition factor to create engaged coherent social teams that work together to win. A special mobile chat is also available to the agent to share tips on how to carry out the missions with other team members.

\subsection{System and Infrastructure}

To monitor electricity consumption, PowerAgent makes use of existing automatic meter reading (AMR) systems in the players' homes. Basically, the AMR systems are connected to the residential central fuse hubs and provide consumption data automatically to the servers at the power companies via a computer network. 


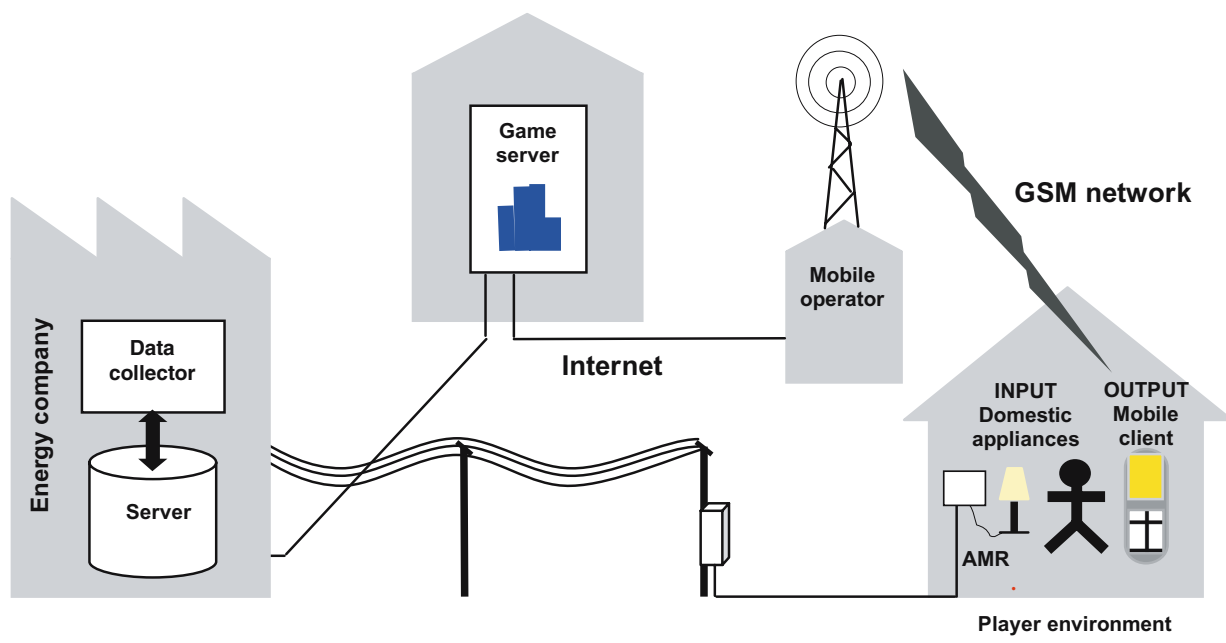

Fig. 2. Infrastructure of PowerAgent

A set of collector daemons relays this information to a central game server that processes and normalizes the data for the game so that all players have the same chance of winning. ${ }^{3}$ Figure 2 shows the infrastructure of the game.

Unfortunately, a deficiency in our prototype is that the data can be delayed up to 12 hours in the power grid due to the underlying implementation of the AMR databases. This means that even though we can monitor each player's consumption on an hourly basis in most cases, the full results might not be accessible until next day. It was necessary to design the game based on those limitations.

The mobile game client that accesses the energy statistics is implemented in Java for SonyEricsson K750 mobiles, and the central game server is implemented in Python. An additional Java-based chat server is also provided in the system to support in-game communication among players.

\subsection{Playing the Game}

Before the beginning of a mission, the game client asks the user to logon to the Agent Network (Fig. 3a). Once logged on, the player is directed to headquarters where Mr. Q is waiting (Fig. 3b). Mr. Q. gives his mission directives verbally, and a countdown clock is started in the background (Fig. 3b). This counter indicates the time remaining until the real-world mission starts at home.

Agent logons are usually requested at $4 \mathrm{PM}$, so that the players can prepare and practice for the upcoming tasks. An important part of the preparations is the abovementioned platform game in which the player can find clues with important information about how to get the best results during the upcoming real-world mission (Fig. 3, d and e).

\footnotetext{
${ }^{3}$ We had to normalize the energy consumption data, because different households use different means of heating, which could have a negative impact on the outcome for some players.
} 

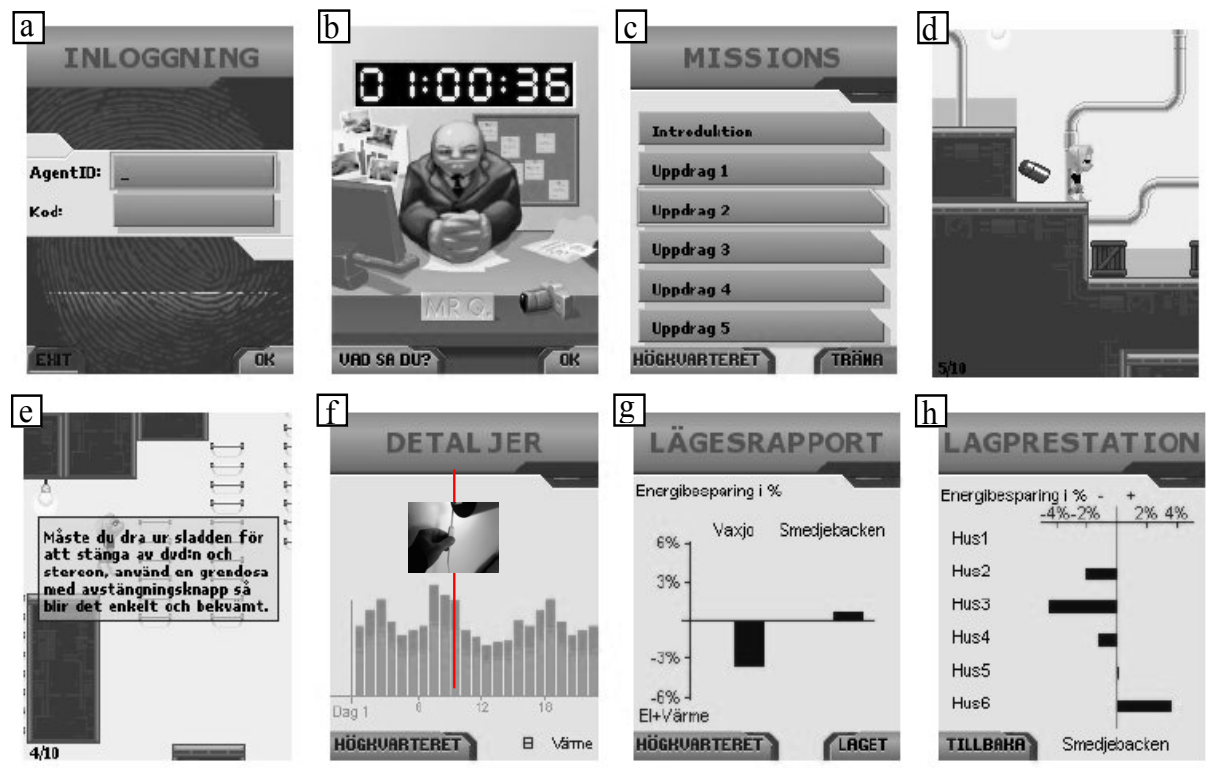

Fig. 3. Screenshots from the PowerAgent

Preparations can also include getting the family to participate in the game session and starting to turn off energy-consuming devices in the home. Other measures can be to check the Internet for information on how to be energy efficient in relation to the specific task. In addition, the player can browse Mr. Q's office when he is not there to access crucial information and chat with other team players.

A typical real-world mission will last for $24-48$ hours, and during the mission the mobile phone notifies the user if something important happens in the game, such as when a sub-task starts or when there is new information available. If the mission lasts for 24 hours or longer, a partial result is presented halfway through the mission. Once a partial or a final result is available, the user is notified and asked to logon to the agent network. Then the player again meets Mr. Q, who provides feedback on both the individual player's work and on the team results. The player can also view individual and team performance statistics (Fig. 3, f, g and h).

Every real agent records his or her mission carefully. Hence, an important task for the energy agent is to document the conservation actions made in their homes. They are using the camera in the mobile phone for this purpose. These pictures are time stamped, mapped to the consumption data, and presented on a timeline after the end of the mission (Fig. 3f). In this way, the pictures serve as reminders of the actions and enable players to get feedback as to whether the things they have done had any effect on the electricity consumption. 


\section{Discussion}

Traditional simulation games for learning have been questioned regarding whether learners are really able to generalize knowledge they acquire in a virtual game world and apply it to real life situations. This is a phenomenon referred to as knowledge transfer, and it is of central interest to researchers in pedagogical psychology. However, when performing the learning tasks in the real world, such as in the PowerAgent game, the mentioned uncertainty becomes, as we shall demonstrate, at least partially obsolete. The question here is not whether the game provides a sufficiently accurate simulation of the real world, but rather if the game supports both cognitive and behavioral learning, and if the acquired knowledge can be remembered and applied in similar situations.

Bandura has suggested that the highest level of learning can be achieved if the modeled behavior can first be symbolically (i.e., cognitively) organized and rehearsed and then overtly enacted [10; cf., 19]. We have attempted to make use of a similar twostage model in PowerAgent. Essentially, PowerAgent can be looked upon as a hybrid game, since it has both virtual and pervasive gaming parts. The underlying idea is to let the users first play a simulation (platform) game on the phone to symbolically learn wanted behaviors, and then let them enact and rehearse these behaviors at home in the family context (the real-world tasks). Thus, in the real missions, the gamers can test the behaviors and get both direct and social feedback on their actions.

The first version of the game - the one presented in this paper-is limited in that the platform game does not show people performing the target behaviors (i.e., modeling via observation). Hence, the platform part of the game could probably be improved significantly by also allowing the players to catch "batteries" that contain video snippets of people illustrating proper energy conservation behaviors to support observational learning and cognitive modeling. Nevertheless, after conclusion of the platform game (using the model available in the present version), we deliberately provide time to prepare for and reflect on the upcoming real-world task. Being able to rehearse game scenarios and create strategies to solve problems are crucial factors that strengthen cognitive learning and lead to a positive outcome that reinforces the target behaviors [10].

We have also included a text chat function to support knowledge sharing among team members, which agrees with the situated learning theories that emphasize social collaboration to solve tasks. Further improvement might be achieved by including the means for video chatting or attaching movies clips in which gamers show each other how they deal with their missions, and that could strengthen social learning and peer modeling. We are currently performing a pilot study of the game at two sites in Scandinavia. Our goal is to investigate the concepts and the playability and to determine how we should empirically evaluate the cognitive and behavioral learning components.

\section{Conclusion}

In this paper, we present PowerAgent, a pervasive game designed to have a positive influence on energy consumption behaviors and activity patterns in the home. The 
principles underlying the design of the game were derived from situated learning and social learning theory. We believe that the two-stage model for learning, in which gamers observe target behaviors in a simulation game and subsequently enact these behaviors in the real world, is a promising persuasion strategy for future pervasive learning games.

Acknowledgements. The research was funded by the Swedish Energy Agency. The authors would like to thank Växjö Energi AB, Smedjebacken Energi \& Vatten, and Mobile Interaction, Stockholm, Sweden.

\section{References}

1. Mayer, R.E. and Wittrock, M.C., Problem-solving transfer. I.D.C. Berliner \& R.C. Calfee (Eds.), Handbook of educational psychology. Macmillan Library Reference USA, Prentice Hall International, New York, N.Y.

2. Gee, J.P.: What video games have to teach us about learning and literacy. Palgrave Macmillan, New York (2003)

3. Ramsden, P.: Student Learning Research: Retrospect and Prospect. Higher Education Research and Development 4(1), 51-69 (1985)

4. Brown, J.S., Collins, A., Duguid, P.: Situated cognition and the culture of learning. Educational Researcher, 32-42 (January-February, 1989)

5. Lave, J., Wenger, E.: Situated learning: Legitimate peripheral participation. Cambridge University Press (1991)

6. Fogg, B.J.: Persuasive Technology, Using Computers to Change What People Think and Do. Morgan Kaufman Publishers, San Francisco (2003)

7. de Vries, P.: Social presence as a conduit to the social dimensions of online trust. In: IJsselsteijn, W., de Kort, Y., Midden, C., Eggen, B., van den Hoven, E. (eds.) PERSUASIVE 2006. LNCS, vol. 3962, pp. 55-59. Springer, Heidelberg (2006)

8. Magerkurth, C., Cheok, A., Mandryk, R.L., Nilsen, T.: Pervasive games: bringing computer entertainment back to the real world. Computers and Entertainment 3(3), 4(2005)

9. Thomas, S.: Pervasive learning games: Explorations of hybrid educational gamescapes. Simulation \& Gaming 37(1), 41-55 (2006)

10. Bandura, A.: Social learning theory. general Learning press, New York (1977)

11. Bandura, A.: Social Cognitive Theory: An agentic perspective. Annual Review of Psychology 52, 1-26 (2001)

12. Electronic Arts Incorporated. Available at http://thesims.ea.com/

13. Botfighters. Last visited (September 30, 2005), http://www.botfighters.com

14. Lindt, I., Ohlenburg, J., Pankoke-Babatz, U., Prinz, W., Ghellal, S.: Combining Multiple Gaming Interfaces in Epidemic Menace. In: Conference on Human Factors in Computing Systems (CHI 2006), Montreal, Canada, April 24-27 (2006)

15. Sotamaa, O.: All The World's A Botfighter Stage: Notes on Location-based Multi-player Gaming. In: Mäyrä, F. (ed.) Computer Games and Digital Cultures: Conference Proceeding. Studies in Information Sciences, University of Tampere, pp. 35-44 (2002) 